\title{
The Impact of Nearshore Eddies on the Design of Marine Environmental Monitoring Sites
}

\author{
WANG Xing, ZHANG Hongliang, LI Baolei \\ North China Sea Environmental Monitoring Center \\ State Oceanic Administration, \\ Shandong Provincial Key Laboratory of Marine Ecology \\ and Environment \& Disaster Prevention and Mitigation \\ Qingdao, China \\ sino1983@sina.com
}

\author{
WU Lunyu \\ First Institute of Oceanography \\ State Oceanic Administration \\ Qingdao, China \\ wuly@ fio.org.cn
}

\begin{abstract}
Design of the in-situ observational sites depends on topography and hydrodynamic characteristics in the observational region, which is significantly important in marine environmental monitoring and evaluation. The tidal motion in Jiaozhou Bay and Aoshan Bay is simulated in this study using the unstructured grid, finite-volume coastal ocean model (FVCOM). With an accurate geometric representation of irregular coastlines and islands and sufficiently high horizontal resolution in narrow channels, FVCOM provides an accurate simulation of the tidal current in the bays and also resolves the strong tidal flushing processes in the narrow channels. There exist many small-scale eddies due to the interaction of tidal flushing and complex coastlines. Small-scale eddies formation will cause inhomogeneous distribution of the marine environmental element, then affects design of the in-situ observational sites. We proposed that design of the in-situ observational sites should consider nearshore eddies in order to conform to reality and enhance environmental protection.
\end{abstract}

Index Terms - marine monitoring, observational sites, eddy, numerical model, FVCOM.

\section{INTRODUCTION}

With the development of Marine Programs, several large marine survey programs have been conducted over the past few decades, such as National Offshore Marine Survey and Assessment, Bohai Stereo Monitoring, and so on. Marine monitoring was significantly enhanced, and more marine workers participated in marine environmental monitoring and evaluation. Design of the in-situ observational sites depends on topography, hydrodynamic characteristics in the observational region, which is significantly important in marine environmental monitoring and evaluation (Shi et al., 2004). The main methods for design of the in-situ observational sites are: (1) uniform distribution in the observational region, (2) a fan-shaped distribution stations from key region due to hydrodynamic characteristics, (3) along the sections vertical to tidal current, and (4) along the sections vertical to isobaths (Shi et al., 2008). Topography and hydrodynamic characteristics in the observational region are significantly important on design of the in-situ observational sites.

We have conducted many numerical simulations in the coastal area and evaluation work in recent years. It can be found that there exists many small-scale eddies due to the interaction of tidal flushing and complex coastlines. Small-scale eddies formation will cause inhomogeneous distribution of the marine environmental element, then affects design of the in-situ observational sites. In order to get typical in-situ observational sites, the effect of small-scale eddies should be considered.

The objectives of the present study is illustrate the influence of the small-scale eddies on the design of the in-situ observational sites based on numerical simulations in the Jiaozhou Bay and Aoshan Bay. The rest of the paper is organized as follows. Section 2 describes the used model and the methodology of eddy identification. Section 3 presents the results of the model on the eddy formation and its influences. Finally, Section 4 summarizes the main results and suggestions.

\section{MODEL AND METHODOLOGY}

\section{FVCOM}

The numerical model used in this study is FVCOM: a prognostic, unstructured grid, finite-volume, free-surface, three-dimensional (3D) primitive equation coastal ocean and estuarine model developed by Chen et al. (2003). FVCOM utilizes a modified Mellor and Yamada level 2.5 (MY-2.5) and Smagorinsky turbulent closure schemes for the default setup of vertical and horizontal mixing, respectively (Mellor and Yamada, 1982). Unlike existing coastal finite-difference and finite-element models, FVCOM solves the hydrostatic primitive equations by calculating fluxes resulting from a discretization of the integral form of these equations on an unstructured triangular mesh. A state-of-the-art-transformation is used to represent the vertical coordinate. This approach not only takes advantage of finite-element methods for grid 
flexibility and finite-difference methods for numerical efficiency but also provides a good numerical representation of momentum, mass, salt, and heat conservation. The detailed description of FVCOM was given in user manual written by Chen et al. [2004] and example of model validations and applications can be viewed directly at the website: http:// codfish.smast.umassd.edu.

The tidal motion in Jiaozhou Bay and Aoshan Bay is simulated using FVCOM. The triangular meshes of the FVCOM computational domain in the region of Jiaozhou Bay and Aoshan Bay are shown in Fig. 1. The maximum horizontal resolution is $\sim 20 \mathrm{~m}$. With an accurate geometric representation of irregular coastlines and islands and sufficiently high horizontal resolution in narrow channels, FVCOM provides an accurate simulation of the tidal current in the bays and also resolves the strong tidal flushing processes in the narrow channels. More detailed description can be seen in Zhao et al. (2006).
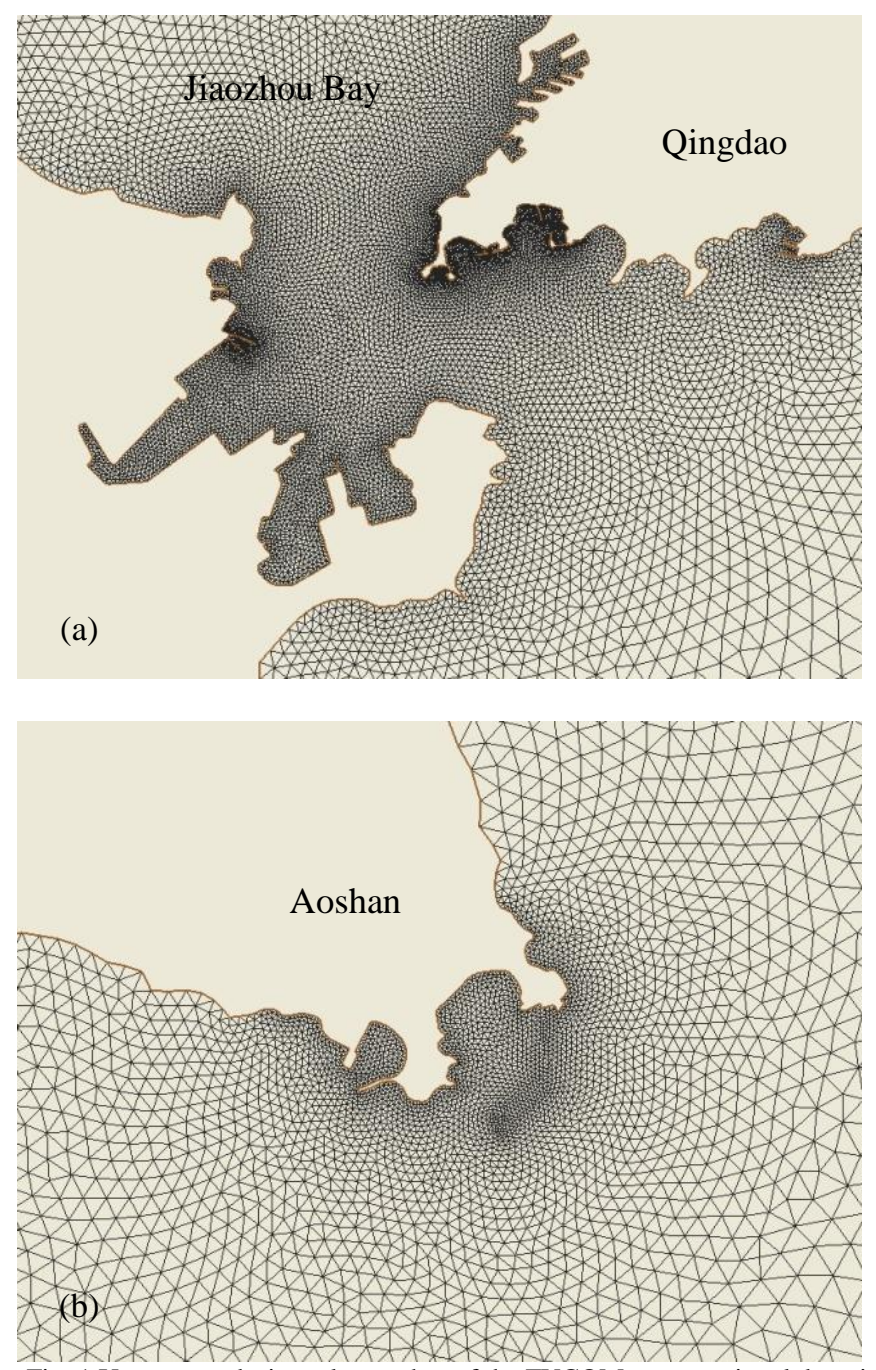

Fig. 1 Unstructured triangular meshes of the FVCOM computational domain with a horizontal resolution of $\sim 20 \mathrm{~m}$ in the region of (a) Jiaozhou Bay and (b) Aoshan Bay.

\section{METHODOLOGY OF EDDY IDENTIFICATION}

Following Jordi et al. (2002), we use the objective criterion of the Okubo-Weiss parameter to identify eddies from SLA maps. The Okubo-Weiss parameter is defined by

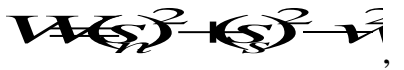

where $s_{n}, s_{s}$, and $w$ are the normal component of strain, the shear component of strain, and the relative vorticity of the flow, defined respectively by

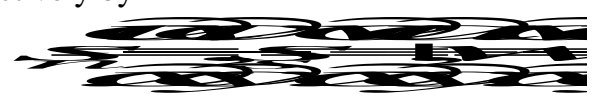

The two-dimensional flow fields can be derived from the model output. This criterion allows us to separate the flow into different regions: a vorticity-dominated region $\left(\mathrm{W}<-0.2 \sigma_{\mathrm{w}}\right)$, a strain-dominated region ( $\mathrm{W}>0.2 \sigma_{\mathrm{w}}$ ), and a background field $\left(|\mathrm{W}| \leq 0.2 \sigma_{\mathrm{w}}\right)$, where $\sigma_{\mathrm{W}}$ is the standard deviation in the whole domain. The region of $\mathrm{W}<-0.2 \sigma_{\mathrm{w}}$ is defined as the eddy core. It is an anticyclonic (cyclonic) eddy when mean sea level anomaly is greater (less) than $0 \mathrm{~cm}$ in this region.

\section{RESULTS AND DISCUSSIONS}

Based on the objective criterion of the Okubo-Weiss parameter, anticyclonic and cyclonic eddies were identified in the Jiaozhou Bay and Aoshan Bay. They are labeled in Fig. 2 and Fig. 3. It can be seen from the distributions of the near-surface tidal current that there exist multiple eddies due to the interaction of tidal flushing and complex coastlines both in Jiaozhou Bay (Fig. 2) and Aoshan Bay (Fig. 3). The formation of these eddies is mainly due to the current separation either at the tip of the coastlines or asymmetric tidal flushing in narrow channels or passages according to Zhao et al. (2006). Based on different period, we separate these eddies into two types: eddy shedding from the tidal flushing, and steady eddies identified from the residual flow field. The influences of two types of eddies on design of the in-situ observational sites will be discussed in next part.

Tide in the Jiaozhou Bay is of regular semi-diurnal type. The mean tidal range is $2.7 \mathrm{~m}$, while the maximum tidal range can reach $6.9 \mathrm{~m}$. Fig. 2 shows the distributions of the near-surface tidal current at the maximum flood, ebb, and residual current in the Jiaozhou Bay, respectively. Both the maximum flood and the maximum ebb appears in the deep channel with the current speed more than $1.5 \mathrm{~m} / \mathrm{s}$, while the maximum residual current is about $0.3 \mathrm{~m} / \mathrm{s}$. On the left (right) side of the current axis, it is easy to generate cyclonic (anticyclonic) eddies. In the center of eddies, the flow speed is small (Fig. 2). It can be inspected that if there is an eddy or eddy rings, the observed current will be obviously different due to the position of the in-situ observational sites. Under these circumstances, the observational data can not represent the real marine environment. Residual flow is an important feature of the hydrodynamic, which has important implications on the study of the marine environment and marine pollution investigations (Hu, 2009). Fig. 2c shows the residual flow field 

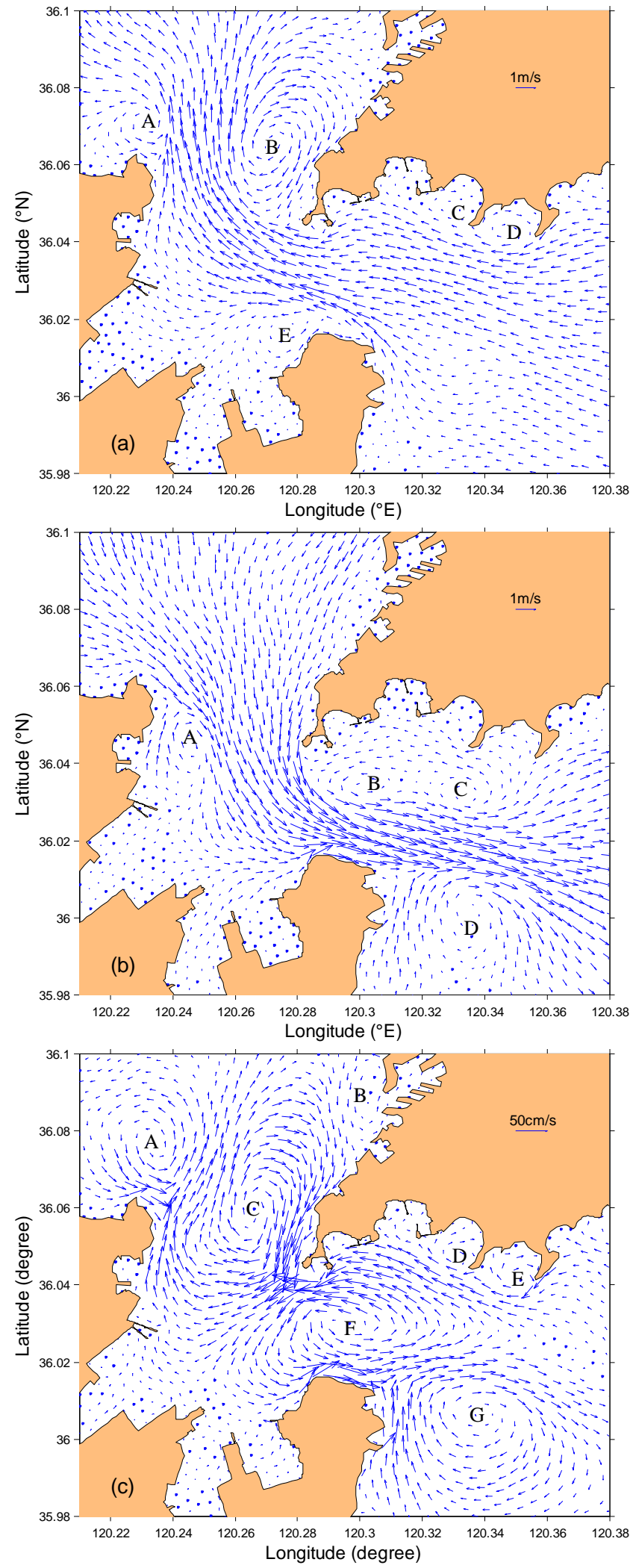

Fig. 2 The distributions of the near-surface tidal current at the maximum flood (a), ebb (b), and residual (c) current in the Jiaozhou Bay.
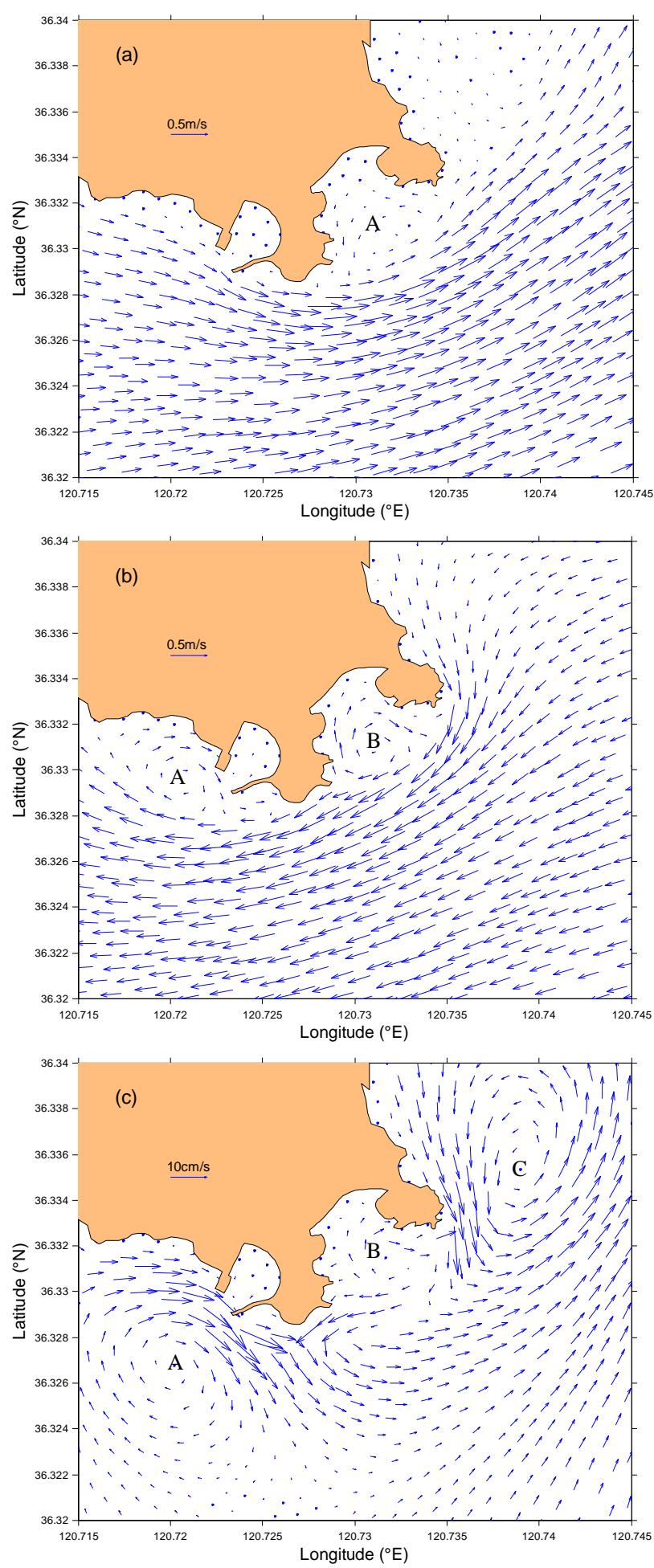

Fig. 3 The distributions of the near-surface tidal current at the maximum flood (a), ebb (b), and residual (c) current in the Aoshan Bay.

of the Jiaozhou Bay. Residual flow is commonly defined as the tidal-cycle average of tidal current. The residual flow described here is the sub-tidal flow that is separated from the tidal current using Foreman's harmonic analysis program. It can be seen from Fig. 2c that multiple eddies formed around headlands, 
convex and concave coastline regions, and channel exits. The water quality conditions are relatively poor (good) if the residual flow direction is out of (in) the Bay. If there is an eddy in the vicinity of the in-situ observational site, the observational data can not represent the real marine environment in this area.

It is similar in the Aoshan Bay. Tide in the Aoshan Bay is also of regular semi-diurnal type. The mean tidal range is $1.2 \mathrm{~m}$, which is smaller than that in the Jiaozhou Bay. Fig. 3 shows the distributions of the near-surface tidal current at the maximum flood, ebb, and residual current in the Aoshan Bay, respectively. Both the maximum flood and the maximum ebb appears in the deep channel with the current speed more than $0.5 \mathrm{~m} / \mathrm{s}$, while the maximum residual current is about $0.15 \mathrm{~m} / \mathrm{s}$. The current speed is much smaller than that in the Jiaozhou Bay. However, multiple eddies formed around headlands, convex and concave coastline regions, which is similar to that in the Jiaozhou Bay. These eddy shedding from tide flushing and steady eddy are significant important on the design of the in-situ observational sites.

In addition to Jiaozhou Bay and Aoshan Bay, there also exist eddies in nearshore areas, such as Bohai Strait and Shidao offshore (Yuan et al., 2007; Xu et al., 2009), due to the current separation either at the tip of the coastlines or asymmetric tidal flushing in narrow channels. Study of eddy distribution is significantly important to improve the representativeness and effectiveness of the monitoring data for marine monitoring workers.

\section{CONCLUSIONS}

Large-area, high-density, long-term marine monitoring takes a lot of manpower and material resources, which is limited by the weather, sea conditions, and environmental conditions. Therefore, in order to reduce the monitoring workload at sea, marine monitoring is often combined with numerical simulation, satellite remote sensing, ground radar to improve the level of monitoring and evaluation. If the number and frequency of monitoring stations are not enough to satisfy the urgent needs, optimizing the layout of the stations is particularly important to obtain more effective data according to the complex hydrodynamic characteristics in the nearshore area.

It can be concluded from the above analysis that multiple eddies often generate around headlands, convex and concave coastline regions due to the interaction of tidal flushing and complex coastlines. So design of the in-situ observational sites should consider nearshore eddies in order to conform to reality and enhance environmental protection. Several suggestions are proposed as follows: (1) if the concerned monitoring scale is greater than the eddy scale, observational site should be set far away from eddies to avoid small-scale disturbances, (2) if the concerned monitoring scale is similar to the eddy scale, distance between observational sites should be set smaller than the radius of eddies in this area, and (3) if the concerned monitoring scale is smaller than the eddy scale, observational site should be set closer as soon as possible, and the monitoring should be conducted during the period of flood tide and ebb tide.

\section{ACKNOWLEDGMENT}

This work was jointly supported by Public science and technology research funds projects of ocean (No. 201005018) and Shandong Provincial Key Laboratory of Marine Ecology and Environment \& Disaster Prevention and Mitigation (No. 2012001).

\section{REFERENCES}

[1] Chen, C., H. Liu, and R. Beardsley, An unstructured grid, finitevolume, three-dimensional, primitive equations ocean model: Application to coastal ocean and estuaries, J. Atmos. Ocean Technol., 2003, 20(1), pp.159-186.

[2] Chen, C., G. Cowles, and R. C. Beardsley, An unstructured grid, finite-volume coastal ocean model: FVCOM user manual, SMAST/UMASSD Tech. Rep., 04-0601, 2009, pp.183.

[3] HU Zhan-ming, Effects of the tide-induced residual current to accumulation of pollutants, Dalian University of Technology Master's degree thesis, 2009, pp.46-49

[4] Jordi, I.-F., G.-L. Emilio and J. Font, Identification of marine eddies from altimetric maps, Journal of Atmospheric and Oceanic Technology, 2002, 20, pp.772-778.

[5] Mellor, G. L., and T. Yamada, Development of a turbulence closuremodel for geophysical fluid problem, Rev. Geophys., 1982, 20, 851- 875 .

[6] SHI Mao-chong, Physical oceanography, Shandong Education Press, 2004, pp. 192-234.

[7] SHI Mao-chong, GAO Guo-ping, BAO Xian-wen, Introduction to marine survey methodology, China Ocean University Press, 2008, pp. 407-413.

[8] XU Dan-ya, Zhao Bao-ren, A Numerical Study on the Mesoscale Anticyclonic Eddy in Qingdao-Shidao Offshore in Winter, Marine Sciences, 2009, NO.2, pp.64-67. (in Chinese with English abstract)

[9] YUAN Yaochu, GUAN Bingxian, Overview of studies on some eddies in the China seas and their adjacent seas, ACTA OCEANOLOGICA SINICA, 2007,Vol. 29, NO.2, pp.1-17. (in Chinese with English abstract)

[10] Zhao, L., C. Chen, and G. Cowles, Tidal flushing and eddy shedding in Mount Hope Bay and Narragansett Bay: An application of FVCOM, J. Geophys. Res., 2006, 111, C10015, doi:10.1029/2005JC003135. 